\title{
A finiteness structure on resource terms
}

\author{
Thomas Ehrhard* \\ Preuves, Programmes, Systèmes \\ CNRS and Université Paris Diderot - Paris 7 \\ Thomas.Ehrhardepps.jussieu.fr
}

October 30, 2018

\begin{abstract}
In our paper "Uniformity and the Taylor expansion of ordinary lambda-terms" (with Laurent Regnier), we studied a translation of lambda-terms as infinite linear combinations of resource lambda-terms, from a calculus similar to Boudol's lambda-calculus with resources and based on ideas coming from differential linear logic and differential lambda-calculus. The good properties of this translation wrt. beta-reduction were guaranteed by a coherence relation on resource terms: normalization is "linear and stable" (in the sense of the coherence space semantics of linear logic) wrt. this coherence relation. Such coherence properties are lost when one considers non-deterministic or algebraic extensions of the lambda-calculus (the algebraic lambda-calculus is an extension of the lambda-calculus where terms can be linearly combined). We introduce a "finiteness structure" on resource terms which induces a linearly topologized vector space structure on terms and prevents the appearance of infinite coefficients during reduction, in typed settings.
\end{abstract}

\section{Introduction}

Denotational semantics and linear logic. Denotational semantics consists in interpreting syntactical objects (programs, proofs) as points in abstract structures (typically, ordered sets with various completeness properties). In this process, the dynamical features of programs are lost, and abstract properties of programs, such as continuity, stability or sequentiality are expressed.

A program, or a proof, is normally a finite object, and its denotation is usually infinite, because it describes all the possible behaviors of the program when applied to all possible arguments. Semantics turns the potential infinity of program dynamics into the actually infinite static description of all its potential behaviors.

Linear logic (LL), which arose from investigations in denotational semantics, sheds a new light on this picture. Whilst being as expressive as intuitionistic logic, LL contains a purely linear fragment which is completely finite in the sense that, during reduction, the size of proofs strictly decreases. For allowing to define and manipulate potentially infinite pieces of proofs/programs, LL introduces new connectives: the exponentials.

Unlike its finite multiplicative-additive fragment, the exponential fragment of LL is strongly asymmetric:

- on one side, there is a promotion rule which allows to introduce the "!" connective and makes a proof duplicable and erasable;

- and on the other side, there are the rules of contraction, weakening and dereliction which allow to duplicate, erase and access to promoted proofs. These rules introduce and allow to perform deductions on the "?" connective, which is the linear dual of "!". Let use call these rules structura 1].

\footnotetext{
${ }^{*}$ This work has been partly funded by the ANR project BLAN07-1 189926 Curry-Howard for Concurrency (CHOCO).

${ }^{1}$ It is not really standard to consider dereliction as structural.
} 
The only infinite rule of LL is promotion. The potentially infinite duplicating power of contraction is not "located" in the contraction rule itself, but in the fact that, for being duplicable by contractions, a proof must be promoted first. This fact can be observed in denotational models but is not clear in the syntax because the structural rules have no other opponents but promotion?

Differential linear logic The situation is quite different in differential LL (and, implicitly, in differential lambdacalculus and its variants), a system that we introduced recently (see [ER03, ER06b, EL09]). In this system, the "?" rules have exact dual rules: there is a cocontraction, a coweakening and a codereliction rules. These rules are logical versions of standard mathematical operations used in elementary differential calculus, whence the name of the system.

So in differential LL we have structural and costructural rules and these rules interact in a completely symmetric and finite way, just as in the multiplicative and additive fragment. Promotion remains apart, as the only truly infinite rule of logic. This fact, which in LL could be observed only in denotational models, can be expressed syntactically in differential LL by means of the Taylor expansion of promotion rules.

Resource lambda-calculus. This operation is more easily understood in the lambda-calculus (see [Tra08] for the connection between lambda-terms and nets in differential LL). Roughly speaking, the ordinary lambda-calculus correspond to the fragment of LL which contains the multiplicative, structural and promotion rules. But we can also consider a lambda-calculus corresponding to the multiplicative, structural and costructural rules: the resource calculus that we introduced in [ER08]. Similar calculi already existed in the literature, such as Boudol's calculi with multiplicities [Bou93] or with resources [BCL99], and also Kfoury's calculi [Kfo00], introduced with different motivations and with different semantic backgrounds. The intuition behind our calculus with resources is as follows.

The first thing to say is that types should be thought of as (topological) vector spaces and not as domains. Consider then a term $t: A \Rightarrow B$ which should be seen as a function from $A$ to $B$. Then imagine that it makes sense to compute the $n$-the derivative of $t$ at the point 0 of the vector space $A$ : it is a function $t^{(n)}(0): A^{n} \rightarrow A$, separately linear in each of its argument, and symmetric in the sense that $t^{(n)}(0)\left(s_{1}, \ldots, s_{n}\right)=t^{(n)}(0)\left(s_{f(1)}, \ldots, s_{f(n)}\right)$ for any permutation $f \in \mathfrak{S}_{n}$ and any tuple $\left(s_{1}, \ldots, s_{n}\right) \in A^{n}$. In our resource calculus, we have an application construction which represents this operation. Given a term $t$ (of type $A \Rightarrow B$ if we are in a typed setting) and a finite number $s_{1}, \ldots, s_{n}$ of terms (of type $A$ ), we can "apply" $t$ to the multiset $S=s_{1} \cdots s_{n}$ (the multiset whose elements are $s_{1}, \ldots, s_{n}$, taking multiplicities into account) and we denote with $\langle s\rangle S$ this operation. We take benefit of the intrinsic commutativity of multisets for implementing the symmetry of the $n$-th derivative. The other constructions of this calculus are standard: we have variables $x, y, \ldots$ and abstractions $\lambda x s$. Redexes are terms of the shape $\langle\lambda x s\rangle S$ and $x$ can have several free occurrences in $s$, which are all linear. When reducing this redex, one does not duplicate $S$. Instead, one splits it into as many pieces as there are occurrences of $x$ in $s$, and since all these occurrences are linear, all these pieces should contain exactly one term. We do that in all possible ways and take the sum of all possible results. When the number of free occurrences of $x$ in $s$ and the size of $S$ do not coincide, the result of this operation is 0 .

For this to make sense, one must have the possibility of adding terms, and this is compatible with the idea that types are vector spaces.

Taylor expansion. Taylor expansion consists in replacing the ordinary application of lambda-calculus with this differential application of the resource calculus. If $M: A \Rightarrow B$ and $N: A$ are terms, then the standard Taylor formula should be

$$
(M) N=\sum_{n=0}^{\infty} \frac{1}{n !} M^{(n)}(0)(\overbrace{N, \ldots, N}^{n})
$$

This leads to the idea of writing any term $M$ as an infinite linear combination of resource terms (with rational coefficients): if $M^{*}$ and $N^{*}$ are such sums, we should have

$$
((M) N)^{*}=\sum_{n=0}^{\infty} \frac{1}{n !}\left\langle M^{*}\right\rangle\left(N^{*}\right)^{n}
$$

\footnotetext{
${ }^{2}$ This picture is not completely faithful because promotion has also to be considered as a "?" rule.
} 
where the power $\left(N^{*}\right)^{n}$ has to be understood in the sense of multiset concatenation, extented to linear combinations of multisets by linearity. Using the fact that all the constructions of the resource calculus should be linear (that is, should distribute over arbitrary linear combinations), formula (1) leads to a definition of $M^{*}$ as a linear combination of resource terms: $M^{*}=\sum_{s \in \Delta} M_{s}^{*} s$ where each $M_{s}^{*}$ is a positive rational number ( $\Delta$ is the set of resource terms): this is the Taylor expansion of $M$.

Taylor expansion looks like denotational semantics: we have transformed a finite program $M$ with a rich, potentially infinite, dynamics into an infinite set (linear combination to be more precise) of more elementary things, the resource terms. The difference wrt. denotational semantics is that these terms have still a dynamics, but this dynamics is completely finite because they belong to the promotion-free fragment of differential linear logic: all terms of our resource calculus, even the non typeable ones, are trivially strongly normalizing. But of course there is no uniform bound on the length of the reductions of the resource terms appearing in the Taylor expansion of a term.

Content. The present article is a contribution to a programme which consists in considering infinite linear combinations of resource terms as generalized lambda-terms. The first point to understand is how beta-reduction can be applied to such infinite linear combinations without introducing infinite coefficients. We initiated this programme in [ER08], defining a binary symmetric, but not reflexive, coherence relation on resource terms (such a coherence relation has also been defined for differential interaction nets in [PT09a]) and showing that, if two terms $s$ and $t$ are coherent and distinct, then their normal forms are disjoint (and hence can be summed). So a first idea is to consider cliques as generalized lambda-terms, and this is sound because the resource terms appearing in the Taylor expansion of a lambda-term are pairwise coherent.

But if we allow linear combinations in the lambda-calculus (as in the differential lambda-calculus for instance, and we speak then of algebraic lambda-calculus 3 , then we cannot expect Taylor expansions to be cliques for that coherence relation. Instead, we equip the set of resource terms with a finiteness structure (in the sense of [Ehr05]) which is defined in such a way that for any "finitary" linear combination $\sum_{s} \alpha_{s} s$ of resource lambda-terms, the sum $\sum_{s} \alpha_{s} a_{s}$ always makes sense, whatever be the choices of $a_{s}$ such that $s$ beta-reduces to $a_{s}$ in the resource lambda-calculus. We prove a soundness theorem, showing that the Taylor expansion of an algebraic lambda-terms is always finitary. This cannot hold however for the untyped algebraic lambda-calculus because we know that this calculus leads to unbounded coefficients during beta-reduction (think of $(\Theta) \lambda x(z+x)$ where $z \neq x$ and $\Theta$ is the Turing fixpoint combinator). So we prove our soundness result for second-order typeable algebraic lambda-terms, by a method similar to Girard's proof of strong normalization of system F in Krivine's very elegant presentation [Kri93]. The method consists in associating with any type a finiteness space (and hence a linearly topologized vector space) whose underlying set (web) is a set of resource terms.

\section{The resource lambda-calculus}

\subsection{The calculus}

The syntax of our resource calculus is defined as follows. One defines first the set $\Delta$ of simple terms and the set $\Delta^{!}$of simple poly-terms.

- If $x$ is a variable then $x \in \Delta$;

- if $s \in \Delta$ and $x$ is a variable then $\lambda x s \in \Delta$;

- if $s \in \Delta$ and $S \in \Delta^{!}$then $\langle s\rangle S \in \Delta$;

- if $s_{1}, \ldots, s_{n} \in \Delta$ then the multiset which consists of the $s_{i} \mathrm{~s}$, denoted in a multiplicative way as $s_{1} \cdots s_{n}$, is an element of $\Delta^{!}$. The empty simple poly-term is accordingly denoted as 1 .

We define the size $\mathrm{S}(s)$ of a simple term $s$ and the size $\mathrm{S}(S)$ of a simple poly-term by induction as follows:

\footnotetext{
${ }^{3}$ There are other algebraizations of the lambda-calculus, we think in particular of the calculus considered by Arrighi and Dowek [AD08] which is quite different from ours because application is right-linear in their setting
} 
- $\mathbf{S}(x)=1$

- $\mathrm{S}(\lambda x s)=1+\mathrm{S}(s)$

- $\mathrm{S}(\langle s\rangle S)=1+\mathrm{S}(s)+\mathrm{S}(S)$

- $\mathrm{S}\left(s_{1} \cdots s_{2}\right)=\mathrm{S}\left(s_{1}\right)+\cdots+\mathrm{S}\left(s_{n}\right)$.

\subsubsection{Extended syntax.}

Given a rig (semi-ring) $R$ and a set $E$, we denote by $R\langle E\rangle$ the set of all formal finite linear combinations of elements of $E$ with coefficients in $R$ : it is the free $R$-module generated by $E$. If $a \in R\langle E\rangle$ and $s \in E, a_{s} \in R$ denotes the coefficient of $s$ in $a$. We also define $R\langle E\rangle_{\infty}$ as the set of all (not necessarily finite) linear combinations of elements of $E$ with coefficients in $R$; we use the same notations as for the elements of $R\langle E\rangle$ and we use $R\langle E\rangle_{(\infty)}$ to denote both modules, to deal with constructions which are applicable in both settings.

The semi-rings that we consider are

- $\mathbb{S}=\{0,1\}$ with $1+1=1$, so that $\mathbb{S}\langle E\rangle=\mathcal{P}_{\text {fin }}(E)$ and $\mathbb{S}\langle E\rangle_{\infty}=\mathcal{P}(E)$;

- $\mathbb{N}$, and then $\mathbb{N}\langle E\rangle$ is the set of all finite multisets of elements of $E$. Given $a \in \mathbb{N}\langle E\rangle$ and $s \in E$ we write $s \in a$ when $a_{s} \neq 0$;

- a field $\mathbf{k}$, and then $\mathbf{k}\langle E\rangle$ is the $\mathbf{k}$-vector space generated by $E$ and $\mathbf{k}\langle E\rangle_{\infty}$ is also a vector space.

Let $a \in R\langle\Delta\rangle_{(\infty)}$, we set $\lambda x a=\sum_{s \in \Delta} a_{s} \lambda x s \in R\langle\Delta\rangle_{(\infty)}$. Given moreover $A \in R\left\langle\Delta^{!}\right\rangle_{(\infty)}$, we set $\langle a\rangle A=\sum_{s \in \Delta, S \in \Delta^{!}} a_{s} A_{S}\langle s\rangle S \in R\langle\Delta\rangle_{(\infty)}$. Last, given $a(1), \ldots, a(n) \in R\langle\Delta\rangle_{(\infty)}$, we define $a(1) \cdots a(n)$ as $\sum_{s(1), \ldots, s(n) \in \Delta} a(1)_{s(1)} \cdots a(n)_{s(n)}(s(1) \cdots s(n)) \in R\left\langle\Delta^{!}\right\rangle_{(\infty)}$. In that formula, remember that $s(1) \cdots s(n)$ is the multiset made of $s(1), \ldots, s(n)$. This formula expresses that we consider multiset concatenation as a product, and so, when extended to linear combinations, a distributivity law must hold.

In particular, given $a \in R\langle\Delta\rangle_{(\infty)}$ and $n \in \mathbb{N}$, we set $a^{n}=\overbrace{a \cdots a}^{n} \in R\left\langle\Delta^{!}\right\rangle_{(\infty)}$. When $R=\mathbf{k}$, we set $a^{!}=$ $\sum_{n \in \mathbb{N}} \frac{1}{n !} a^{n} \in \mathbf{k}\left\langle\Delta^{!}\right\rangle_{\infty}$ (this sum always makes sense, and we require $R=\mathbf{k}$ to give a meaning to $1 / n$ !). For $e \subseteq \Delta$ (that is $e \in \mathbb{S}\langle\Delta\rangle_{\infty}$ ), we set $e^{!}=\mathcal{M}_{\text {fin }}(e) \subseteq \Delta$ !

So all the constructions of the syntax can be applied to arbitrary linear combinations of simple terms, giving rise to combinations of simple terms.

\subsubsection{Differential substitution}

Given $s \in \Delta$ and $S \in \Delta^{!}$, and given a variable $x$, we define the differential substitution $\partial_{x}(s, S)$ as 0 if the number of free occurrences of $x$ in $s$ is different from $n$, and as $\sum_{f \in \mathfrak{S}_{n}} s\left[s_{f(1)} / x_{1}, \ldots, s_{f(n)} / x_{n}\right]$ otherwise, where $S=$ $s_{1} \cdots s_{n}, x_{1}, \ldots, x_{n}$ are the $n$ occurrences of $x$ in $s$ and $\mathfrak{S}_{n}$ is the group of permutations on $\{1, \ldots, n\}$.

Given $s \in \Delta$ and $S_{1}, \ldots, S_{n} \in \Delta^{!}$and pairwise distinct variables $x_{1}, \ldots, x_{n}$ which do not occur free in the $S_{i}$ 's, we define more generally the parallel differential substitution $\partial_{x_{1}, \ldots, x_{n}}\left(s, S_{1}, \ldots, S_{n}\right)$ : the definition is similar (the sum is indexed by tuples $\left(f_{1}, \ldots, f_{n}\right)$ where $f_{i}$ is a permutation on the free occurrences of $x_{i}$ in $\left.s\right)$.

This operation must be extended by linearity. Given $a \in R\langle\Delta\rangle_{(\infty)}$ and $A \in R\left\langle\Delta^{!}\right\rangle_{(\infty)}$, we set

$$
\partial_{x}(a, A)=\sum_{s \in \Delta, S \in \Delta !} a_{s} A_{S} \partial_{x}(s, S) \in R\langle\Delta\rangle_{(\infty)}
$$

and we define similarly $\partial_{x_{1}, \ldots, x_{n}}\left(a, A_{1}, \ldots, A_{n}\right) \in R\langle\Delta\rangle_{(\infty)}$. It is not obvious at first sight that this sum is well defined in the infinite case. This results from Lemma 6 (see below). 


\subsubsection{The reduction relations}

Given two sets $E$ and $F$ and a relation $\rho \subseteq E \times \mathbb{N}\langle F\rangle$, we define a relation $\mathbb{N}\langle\rho\rangle \subseteq \mathbb{N}\langle E\rangle \times \mathbb{N}\langle F\rangle$ as follows: we say that $(a, b) \in \mathbb{N}\langle\rho\rangle$ if there are $\left(s_{1}, a_{1}\right), \ldots,\left(s_{n}, a_{n}\right) \in \rho$ such that $s_{1}+\cdots+s_{n}=a$ and $b_{1}+\cdots+b_{n}=b$.

The one step reduction relations $\beta_{\Delta}^{1} \subseteq \Delta \times \mathbb{N}\langle\Delta\rangle$ and $\beta_{\Delta^{!}}^{1} \subseteq \Delta^{!} \times \mathbb{N}\left\langle\Delta^{!}\right\rangle$are defined as follows.

- $x \beta_{\Delta}^{1} b$ never holds;

- $\lambda x s \beta_{\Delta}^{1} b$ if $b=\lambda x a$ with $s \beta_{\Delta}^{1} a$;

- $s_{1} \cdots s_{n} \beta_{\Delta !}^{1} B$ if, for some $i, s_{i} \beta_{\Delta}^{1} b_{i}$ and $B=s_{1} \cdots b_{i} \cdots s_{n}$;

- $\langle s\rangle S \beta_{\Delta}^{1} b$ in one of the following situations

- $s \beta_{\Delta}^{1} a$ and $b=\langle a\rangle S$;

- $S \beta_{\Delta !}^{1} A$ and $b=\langle s\rangle A$;

- $s=\lambda x t$ and $b=\partial_{x}(t, S)$.

Lemma 1 Let $s \in \Delta$ and $b \in \mathbb{N}\langle\Delta\rangle$. If $s \beta_{\Delta}^{1} b$, then, for any $t_{1}, t_{2} \in b$, one has $\mathrm{S}\left(t_{1}\right)=\mathrm{S}\left(t_{2}\right)<\mathrm{S}(s)$.

The proof is straightforward (simple case inspection).

Let $\beta_{\Delta}^{0,1}=\mathbb{N}\left\langle\{(s, s) \mid s \in \Delta\} \cup \beta_{\Delta}^{1}\right\rangle$ and $\beta_{\Delta !}^{0,1}=\mathbb{N}\left\langle\left\{(S, S) \mid s \in \Delta^{!}\right\} \cup \beta_{\Delta^{!}}^{1}\right\rangle$. These are reflexive reduction relations on $\mathbb{N}\langle\Delta\rangle$ and $\mathbb{N}\left\langle\Delta^{!}\right\rangle$respectively. More explicitly, we have $a \beta_{\Delta}^{0,1} b$ if one can write $a=s_{1}+\cdots+s_{n}+a^{\prime}$ and $b=b_{1}+\cdots+b_{n}+a^{\prime}$ with $s_{i} \beta_{\Delta}^{1} b_{i}$ for $i=1, \ldots, n$, and similarly for $\beta_{\Delta !}^{0,1}$.

Finally we denote with $\beta_{\Delta}$ and $\beta_{\Delta}$ ! respectively the transitive closures of these relations.

Lemma 2 Let $s, t \in \Delta$ and $x$ is a variable which occurs free exactly once in $s$. If $s \beta_{\Delta}^{1}$ a then $s[t / x] \beta_{\Delta}^{1} a[t / x]$ and ift $\beta_{\Delta}^{1} b$ then $s[t / x] \beta_{\Delta}^{1} s[b / x]$.

Lemma 3 If $s \beta_{\Delta}^{1}$ a then $\partial_{x}(s, S) \mathbb{N}\left\langle\beta_{\Delta}^{1}\right\rangle \partial_{x}(a, S)$. If $S \beta_{\Delta}^{1} A$ then $\partial_{x}(s, S) \mathbb{N}\left\langle\beta_{\Delta}^{1}\right\rangle \partial_{x}(s, A)$.

These two lemmas are proved by straightforward inductions.

The reduction relation $\beta_{\Delta}$ on $\mathbb{N}\langle\Delta\rangle$ has good properties: it is strongly normalizing, confluent (see [ER03, Vau05, PT09b]). Given $s \in \Delta$, we denote by $\operatorname{NF}(s)$ the unique normal form of $s$, which is an element of $\mathbb{N}\langle\Delta\rangle$.

\subsubsection{Examples of reduction}

Of course $\langle\lambda x x\rangle y \beta_{\Delta} y$, but if the identity is applied to a multiset of size $\neq 1$, the result is $0:\langle\lambda x x\rangle 1 \beta_{\Delta} 0$ and $\langle\lambda x x\rangle y^{2} \beta_{\Delta} 0$ (where $y^{2}$ is the multiset which contains twice the variable $y$; this notation is compatible with the distributivity laws of 1.1.1).

Similarly, the term $\langle x\rangle x^{2}$ contains 3 occurrences of $x$ (it is sensible to say that it is of degree 3 in $x$ ). So $\left\langle\lambda x\langle x\rangle x^{2}\right\rangle S \beta_{\Delta} 0$ if the size of $S$ is $\neq 3$. And we have $\left\langle\lambda x\langle x\rangle x^{2}\right\rangle\left(y^{2} z\right) \beta_{\Delta} \partial_{x}\left(\langle x\rangle x^{2}, y^{2} z\right)=4\langle y\rangle y z+2\langle z\rangle y^{2}$. As a last example we have $\langle\lambda x\langle\langle x\rangle x\rangle x\rangle\left(y^{2} z\right) \beta_{\Delta} \partial_{x}\left(\langle x\rangle x^{2}, y^{2} z\right)=2\langle\langle y\rangle z\rangle y+2\langle\langle y\rangle y\rangle z+2\langle\langle z\rangle y\rangle y$.

\subsubsection{An order relation on simple terms and poly-terms.}

Let us define an order relation on simple terms. Given $s, t \in \Delta$, we write $t \leq s$ if there exists $a \in \mathbb{N}\langle\Delta\rangle$ such that $s \beta_{\Delta} a$ and $t \in a$. Given $s \in \Delta$, we use $\downarrow s=\{t \in \Delta \mid t \leq s\}$ and $\uparrow s=\{t \in \Delta \mid t \geq s\}$. We define similarly an order relation on poly-terms and introduce similar notations: $T \leq S, \uparrow S$ and $\downarrow S$.

Lemma 4 For any $s \in \Delta$, the set $\downarrow s$ is finite.

Proof. By Lemma 1 and König's lemma. 


\subsection{Two technical lemmas}

Lemma 5 Let y be a variable and $S_{1}, \ldots, S_{n} \in \Delta !$ which do not contain free the variable $y$ and let $v=\left\langle\cdots\left\langle\langle y\rangle S_{1}\right\rangle S_{2} \cdots\right\rangle S_{n}$. Let $s \in \Delta, S \in \Delta !, x$ be a variable. Let $t \in \Delta$ be such that $t \leq v[\langle\lambda x s\rangle S / y]$. Then one of the the two following cases arises:

- either $t=v^{\prime}\left[\left\langle\lambda x s^{\prime}\right\rangle S^{\prime} / y\right]$ with $v^{\prime} \leq v, s^{\prime} \leq s$ and $S^{\prime} \leq S$

- or $t \leq v[u / y]$ for some $u \in \partial_{x}(s, S)$.

Proof. By induction on $\mathrm{S}(v)+\mathrm{S}(s)+\mathrm{S}(S)$. Let $b \in \mathbb{N}\langle\Delta\rangle$ be such that $v[\langle\lambda x s\rangle S / y] \beta_{\Delta} b$ and $t \in b$. Consider the first reduction step of this reduction. Four cases are possible, because of the particular shape of $v$.

First case: the reduction occurs in $s$. That is $s \beta_{\Delta}^{1} a$ for some $a \in \mathbb{N}\langle\Delta\rangle$ and the reduction $v[\langle\lambda x s\rangle S / y] \beta_{\Delta} b$ splits in $v[\langle\lambda x s\rangle S / y] \beta_{\Delta}^{1} v[\langle\lambda x a\rangle S / y] \beta_{\Delta} b$. Since $t \in b$, one can find some $u \in \Delta$ with $u \in a$ such that $t \leq v[\langle\lambda x u\rangle S / y]$. Since $\mathrm{S}(u)<\mathrm{S}(s)$, the inductive hypothesis applies and so there are two cases.

- Either we have $t=v^{\prime}\left[\left\langle\lambda x u^{\prime}\right\rangle S^{\prime} / y\right]$ with $v^{\prime} \leq v, u^{\prime} \leq u$ and $S^{\prime} \leq S$ and we conclude because $u<s$.

- Or $t \leq v[w / y]$ with $w \in \Delta$ such that $w \in \partial_{x}(u, S)$. Since $u \in a$ and $w \in \partial_{x}(u, S)$, we have $w \in \partial_{x}(a, S)$. But $\partial_{x}(s, S) \mathbb{N}\left\langle\beta_{\Delta}^{1}\right\rangle \partial_{x}(a, S)$ by Lemma 3 and hence there exists $w_{0} \in \partial_{x}(s, S)$ such that $w<w_{0}$. Hence we have $v[w / y]<v\left[w_{0} / y\right]$ by Lemma 2 and we conclude by transitivity.

The second case, where the reduction occurs in $S$ is similar.

Third case: the reduction occurs in $v$. That is $v \beta_{\Delta}^{1} c \in \mathbb{N}\langle\Delta\rangle$ and the reduction $v[\langle\lambda x s\rangle S / y] \beta_{\Delta} b$ splits in $v[\langle\lambda x s\rangle S / y] \beta_{\Delta}^{1} c[\langle\lambda x s\rangle S / y] \beta_{\Delta} b$. Since $t \in b$, one can find some $w \in c$ such that $t \leq w[\langle\lambda x s\rangle S / y]$. Since $\mathrm{S}(w)<\mathrm{S}(v)$, the inductive hypothesis applies and so there are two cases.

- Either $t=w^{\prime}\left[\left\langle\lambda x s^{\prime}\right\rangle S^{\prime} / y\right]$ with $w^{\prime} \leq w, s^{\prime} \leq s$ and $S^{\prime} \leq S$ and we conclude because $w \leq v$.

- Or $t \leq w[u / y]$ for some $u \in \partial_{S}(s, x)$. We conclude by Lemma 2 because $w<v$.

Last case: the reduction $v[\langle\lambda x s\rangle S / y] \beta_{\Delta} b$ splits in $v[\langle\lambda x s\rangle S / y] \beta_{\Delta}^{1} v\left[\partial_{x}(s, S) / y\right] \beta_{\Delta} b$ and we conclude immediately that there exists $u \in \partial_{x}(s, S)$ such that $t \leq v[u / x]$.

Lemma 6 Let $s \in \Delta$. There are only finitely many pairs $(t, T) \in \Delta \times \Delta^{!}$such that $s \in \partial_{x}(t, T)$.

Proof. (Sketch) The intuition is clear and can easily be formalized. For building $(t, T)$, one must choose some $n \in \mathbb{N}$, and then $n$ pairwise disjoin 4 sub-terms $t_{1}, \ldots, t_{n}$ of $s$. Then $t$ is obtained by replacing these sub-terms by $x$ in $s$, and $T=t_{1} \cdots t_{n}$. There are only finitely many ways of choosing such a tuple $\left(n, t_{1}, \ldots, t_{n}\right)$.

\section{Finiteness spaces}

We recall some basic material on finiteness spaces. Given a set $I$ and a collection $\mathcal{F}$ of subsets of $I$, we define

$$
\mathcal{F}^{\perp}=\left\{e^{\prime} \subseteq I \mid \forall e \in \mathcal{F} e \cap e^{\prime} \text { is finite }\right\} .
$$

A finiteness space is a pair $X=(|X|, \mathrm{F}(X))$ where $|X|$ is a set (the web of $X)$ and $\mathrm{F}(X) \subseteq \mathcal{P}(|X|)$ satisfies $\mathrm{F}(X)^{\perp \perp} \subseteq \mathrm{F}(X)$ (the other inclusion being always true). The following properties follow immediately from this definition: if $e \subseteq|X|$ is finite then $e \in \mathrm{F}(X)$; if $e \in \mathrm{F}(X)$ and $f \subseteq e$ then $f \in \mathrm{F}(X)$; if $e_{1}, e_{2} \in \mathrm{F}(X)$ then $e_{1} \cup e_{2} \in \mathrm{F}(X)$.

\footnotetext{
${ }^{4}$ None of these terms can be a sub-term of another one.
} 
Vector space. Let $\mathbf{k}$ be a field. Given $a \in \mathbf{k}^{|X|}$, let $\operatorname{Supp}(a)=\left\{s \in|X| \mid a_{s} \neq 0\right\}$ (the support of $a$ ). We set $\mathbf{k}\langle X\rangle=\left\{a \in \mathbf{k}^{|X|} \mid \operatorname{Supp}(a) \in \mathrm{F}(X)\right\}$. This set is a k-vector space, addition and scalar multiplication being defined pointwise.

Topology. Given $e^{\prime} \in \mathrm{F}(X)^{\perp}$, let $\mathrm{V}_{0}\left(e^{\prime}\right)=\left\{a \in \mathbf{k}\langle X\rangle \mid \operatorname{Supp}(a) \cap e^{\prime}=\emptyset\right\}$ : this is a linear subspace of $\mathbf{k}\langle X\rangle$. A subset $\mathcal{V}$ of $\mathbf{k}\langle X\rangle$ is open if, for all $a \in \mathcal{V}$ there exists $e^{\prime} \in \mathrm{F}(X)^{\perp}$ such that $a+\mathrm{V}_{0}\left(e^{\prime}\right) \subseteq \mathcal{V}$. This defines a topology for which one checks easily that addition and scalar multiplication are continuous ( $\mathrm{k}$ being equipped with the discrete topology). Actually $\mathbf{k}\langle X\rangle$ is a linearly topologized vector space in the sense of [Lef42]: the topology is generated by neighborhoods of 0 which are linear subspaces (for instance, the $\mathrm{V}_{0}\left(e^{\prime}\right)$ we introduced above). This topology is Hausdorff: for any $a \in \mathbf{k}\langle X\rangle$, if $a \neq 0$ one cant find a (linear) neighborhood of 0 which does not contain $a$. In particular, the specialization ordering is discrete (this is not a topology "à la Scott").

Convergence and completeness. A net of $\mathbf{k}\langle X\rangle$ if a family $(a(\gamma))_{\gamma \in \Gamma}$ of elements $\mathbf{k}\langle X\rangle$ indexed by a directed set $\Gamma$. Such a net converges to $a \in \mathbf{k}\langle X\rangle$ if, for any open linear subspace $\mathcal{V}$ of $\mathbf{k}\langle X\rangle$ there is $\gamma \in \Gamma$ such that $\forall \delta \in \Gamma \delta \geq \gamma \Rightarrow a(\delta)-a \in \mathcal{V}$. If this holds, $a$ is unique $\left(\mathbf{k}\langle X\rangle\right.$ is Hausdorff). A net $(a(\gamma))_{\gamma \in \Gamma}$ is Cauchy if for any open linear subspace $\mathcal{V}$ of $\mathbf{k}\langle X\rangle$, there exists $\gamma \in \Gamma$ such that $\forall \delta \in \Gamma \delta \geq \gamma \Rightarrow a(\delta)-a(\gamma) \in \mathcal{V}$. Using crucially the fact that $\mathrm{F}(X)=\mathrm{F}(X)^{\perp \perp}$, one can prove that any Cauchy net converges ( $\mathbf{k}\langle X\rangle$ is complete).

\section{The basic finiteness structure}

We set

$$
\begin{aligned}
\mathcal{N}_{1} & =\{\uparrow s \mid s \in \Delta\}^{\perp} \\
& =\{e \subseteq \Delta \mid \forall s \in \Delta \quad e \cap \uparrow s \text { is finite }\} .
\end{aligned}
$$

One defines similarly $\mathcal{N}_{1}{ }^{!} \subseteq \mathcal{P}\left(\Delta^{!}\right)$as $\mathcal{N}_{1}{ }^{!}=\left\{E \subseteq \Delta^{!} \mid \forall S \in \Delta^{!} \quad E \cap \uparrow S\right.$ is finite $\}$. This defines finiteness structures on $\Delta$ and $\Delta !$. We consider therefore $\left(\Delta, \mathcal{N}_{1}\right)$ as a finiteness space that we simply denote as $\mathcal{N}_{1}$. To get a better grasp of the topology of the vector space $\mathbf{k}\left\langle\mathcal{N}_{1}\right\rangle$, we must make a first observation. We express everything for $\Delta$ for notational convenience, but obviously what we do can be transposed to $\Delta$ ! without any difficulty.

Lemma 7 A subset $e^{\prime}$ of $\Delta$ belongs to $\mathcal{N}_{1}{ }^{\perp}$ iff there are finitely many elements $s_{1}, \ldots, s_{n} \in \Delta$ such that

$$
e^{\prime} \subseteq \uparrow s_{1} \cup \cdots \cup \uparrow s_{n}=\uparrow\left\{s_{1}, \ldots, s_{n}\right\} .
$$

Proof. The "if" part is trivial, let us check the "only if" part. The only property of the order relation on simple terms that we need is the fact that each set $\downarrow s$ is finite (Lemma 4).

Assume that there exists $e^{\prime} \in \mathcal{N}_{1}{ }^{\perp}$ such that $e^{\prime} \subseteq \uparrow\left\{s_{1}, \ldots, s_{n}\right\}$ never holds. The set $e^{\prime}$ cannot be empty, so let $u_{1} \in e^{\prime}$. Since $\downarrow u_{1}$ is finite, we cannot have $e^{\prime} \subseteq \uparrow \downarrow u_{1}$. So let $u_{2} \in e^{\prime} \backslash \uparrow \downarrow u_{1}$. Again, $\downarrow u_{2}$ being finite, we cannot have $e^{\prime} \subseteq \uparrow \downarrow u_{1} \cup \uparrow \downarrow u_{2}$. In that way, we construct an infinite sequence $u_{1}, u_{2} \ldots$ of elements of $e^{\prime}$ such that for each $i, u_{i+1} \in e^{\prime} \backslash\left(\uparrow \downarrow u_{1} \cup \cdots \cup \uparrow \downarrow u_{i}\right)$; in particular, the $u_{i}$ 's are pairwise distinct, but we can say better: let $i<j$ and assume that $\downarrow u_{i} \cap \downarrow u_{j} \neq \emptyset$. Then $u_{j} \in \uparrow \downarrow u_{i}$ and this is impossible. Let us set $e=\left\{u_{1}, u_{2}, \ldots\right\}$. For any $s \in \Delta$, it follows from the disjointness of the sets $\downarrow u_{i}$ that $e \cap \uparrow s$ has at most one element and is therefore finite, so that $e \in \mathcal{N}_{1}$. But $e$ has an infinite intersection with $e^{\prime}$ (namely $e$ ), and this contradicts our hypothesis that $e^{\prime} \in \mathcal{N}_{1}{ }^{\perp}$.

Therefore the topology of $\mathbf{k}\left\langle\mathcal{N}_{1}\right\rangle$ is generated by the basic neighborhoods $\mathrm{V}\left(s_{1}, \ldots, s_{n}\right)=\left\{u \in \mathbf{k}\left\langle\mathcal{N}_{1}\right\rangle \mid\right.$ $\left.\operatorname{Supp}(u) \cap \uparrow s_{1}=\cdots=\operatorname{Supp}(u) \cap \uparrow s_{n}=\emptyset\right\}$, where $s_{1}, \ldots, s_{n}$ is an arbitrary finite family of elements of $\Delta$. Observe that these $s_{i}$ 's can be assumed to be minimal in $\Delta$. An element $s$ of $\Delta$ is minimal for the order relation we have defined iff $s$ is normal, or reduces only to 0 . A typical non-normal minimal term is $\langle\lambda x y\rangle$, where $y$ and $z$ are distinct variables.

The main purpose of these definitions is to give meaning to a normalization function on vectors. Consider indeed an arbitrary linear combinations of resource lambda-terms, $a=\sum_{a \in \Delta} a_{s} s \in \mathbf{k}\langle\Delta\rangle_{\infty}$. We would like to set $\operatorname{NF}(a)=$ 
$\sum_{s \in \Delta} a_{s} \mathrm{NF}(s)$. But there could perfectly exist normal elements $s_{0} \in \Delta$ such that, for infinitely many $s \in \Delta$, $s_{0} \in \mathrm{NF}(s)$ and $a_{s} \neq 0$. If this is the case, we cannot normalize $a$ because infinite sums are not allowed in $\mathbf{k}$ which is an arbitrary field 5 . As a typical example of this situation, consider $a=x+\langle\lambda x x\rangle x+\langle\lambda x x\rangle(\langle\lambda x x\rangle x)+\cdots$ All the terms of this sum reduce to the same term $x$ and hence $\operatorname{NF}(a)$ is not defined.

Proposition 8 The map NF given by $\mathrm{NF}(a)=\sum_{s \in \Delta} a_{s} \mathrm{NF}(s)$ is well defined, linear and continuous from the topological vector space $\mathbf{k}\left\langle\mathcal{N}_{1}\right\rangle$ to itself.

Proof. Given $s \in \Delta$, we have $\operatorname{Supp}(\operatorname{NF}(s)) \subseteq \downarrow s$. So, since $\operatorname{Supp}(a) \in \mathcal{N}_{1}$, for any $s_{0} \in \Delta_{0}$, there are only finitely many $s \in \operatorname{Supp}(a)$ such that $s_{0} \in \operatorname{Supp}(\operatorname{NF}(s))$. So the sum above makes sense, it can be written

$$
\mathrm{NF}(a)=\sum_{s_{0} \in \Delta_{0}}\left(\sum_{\substack{s \in \operatorname{Supp}(a) \\ s_{0} \in \downarrow s}} a_{s} \operatorname{NF}(s)_{s_{0}}\right) s_{0} .
$$

All the elements of $\operatorname{Supp}(\mathrm{NF}(a))$ being minimal, this set obviously belongs to $\mathcal{N}_{1}$.

The map NF defined in that way is obviously linear, we must just check that it is continuous at 0 but this is easy; indeed, if $V=\mathrm{V}\left(s_{1}, \ldots, s_{n}\right)$ is a basic neighborhood of 0 then, by definition of $\mathrm{V}\left(s_{1}, \ldots, s_{n}\right)$, if $t \in \Delta$ satisfies $t \in V$, this means that $t \notin \uparrow s_{i}$ for each $i$, and hence for no $i$ we can have $s_{i} \in \operatorname{NF}(t)$. Therefore $\operatorname{NF}(t) \in V$.

We can also extend the $\beta_{\Delta}^{0,1}$ reduction relation to $\mathbf{k}\left\langle\mathcal{N}_{1}\right\rangle$ in a completely "free 6 ", way. Indeed let $a \in \mathbf{k}\left\langle\mathcal{N}_{1}\right\rangle$. If one writes $a=\sum_{i \in \mathbb{N}} \alpha_{i} s_{i}$ with $s_{i} \in \Delta$ and with the sole restriction (for this sum to make sense at all) that for each $s \in \Delta$ there are only finitely $i$ 's such that $s_{i}=s$ and if, for each $i \in \mathbb{N}$, one chooses arbitrarily $a(i) \in \mathbb{N}\langle\Delta\rangle$ such that $s_{i} \beta_{\Delta}^{0,1} a(i)$, then the sum $b=\sum_{i \in \mathbb{N}} \alpha_{i} a(i)$ always makes sense, and belongs to $\mathbf{k}\left\langle\mathcal{N}_{1}\right\rangle$ (these facts result from the very definition of $\mathcal{N}_{1}$ ). In that case we write $a \beta_{\Delta}^{0,1} b$, and we denote by $\beta_{\Delta}$ the transitive closure of $\beta_{\Delta}^{0,1}$.

Proposition 9 The relation $\beta_{\Delta}$ is confluent on $\mathbf{k}\left\langle\mathcal{N}_{1}\right\rangle$.

Proof. (Sketch) Use the confluence of $\beta_{\Delta}^{1}$ on $\mathbb{N}\langle\Delta\rangle$ and the following observation: given two finite families $\left(\alpha_{i}\right)_{i \in I}$ and $\left(\beta_{j}\right)_{j \in J}$ of elements of $\mathbf{k}$ such that $\sum \alpha_{i}=\sum \beta_{j}$, one can find a family $\left(\gamma_{i, j}\right)_{i \in I, j \in J}$ of elements of $\mathbf{k}$ such that $\forall i \alpha_{i}=\sum_{j} \gamma_{i, j}$ and $\forall j \beta_{j}=\sum_{i} \gamma_{i, j}$.

One has to be aware that this "reduction" relation has strange properties and can hardly be expected to normalize in a standard sense. For instance if $s \beta_{\Delta}^{1} a_{1}$ and $s \beta_{\Delta}^{1} a_{2}$ where $a_{1}, a_{2} \in \mathbb{N}\langle\Delta\rangle$ are distinct, then $0=s-s \beta_{\Delta} a_{1}-a_{2} \neq 0$ and the reduction can go on after that. See [Vau07, Vau08] for more explanations. It makes sense nevertheless to define the associated equivalence relation (the symmetric closure of $\beta_{\Delta}$ ) that we denote as $=_{\Delta}$.

Proposition 10 Let $a, b \in \mathbf{k}\langle\Delta\rangle$ be such that $a={ }_{\Delta} b$. Then $\mathrm{NF}(a)=\mathrm{NF}(b)$.

Proof. It suffices to show that $a \beta_{\Delta}^{0,1} b \Rightarrow \mathrm{NF}(a)=\mathrm{NF}(b)$ and this is easy because $s \beta_{\Delta}^{1} c \Rightarrow \mathrm{NF}(s)=\mathrm{NF}(c)$.

The converse implication does not hold because reducing an element $a \in \mathbf{k}\langle\Delta\rangle$ to $\mathrm{NF}(a)$ can require an infinite number of $\beta_{\Delta}^{0,1}$ steps. But one can always exhibit sequences $a=a(1) \beta_{\Delta}^{0,1} a(2) \beta_{\Delta}^{0,1} a(3) \cdots$ with $\lim _{n \rightarrow \infty} a(n)=$ $\mathrm{NF}(a)$ (in the sense of the topology of $\mathbf{k}\left\langle\mathcal{N}_{1}\right\rangle$ ).

Remark: It is not difficult to see that, given a finiteness space $X$, the topological space $\mathbf{k}\langle X\rangle$ is metrizable (ie. its topology can be defined by a distance) iff there exists an increasing sequence $\left(e^{\prime}(n)\right)_{n \in \mathbb{N}}$ of elements of $\mathrm{F}(X)^{\perp}$ such that $\forall e^{\prime} \in \mathrm{F}(X)^{\perp} \exists n \in \mathbb{N} e^{\prime} \subseteq e^{\prime}(n)$. It is also interesting to observe that, when interpreting linear logic in finiteness spaces (see [Ehr05]), one builds quite easily spaces which have not this property: for instance the interpretation of !?1 (the formula 1 being interpreted by the finiteness space $(\{*\},\{\emptyset,\{*\}\}))$ is not metrizable.

\footnotetext{
${ }^{5}$ Of course, one could also consider infinite sums if the coefficients were real or complex numbers but this will be the object of further studies.

${ }^{6}$ In the sense that each summand can be reduced independently from the others.
} 
So the space $\mathbf{k}\left\langle\mathcal{N}_{1}\right\rangle$ is metrizable: choose an enumeration $s_{1}, s_{2}, \ldots$ of $\Delta$ and, given $a, a^{\prime} \in \mathbf{k}\left\langle\mathcal{N}_{1}\right\rangle$, define $d\left(a, a^{\prime}\right)=0$ if $a=a^{\prime}$, and $d\left(a, a^{\prime}\right)=2^{-n}$ where $n$ is the least integer such that $\uparrow s_{n} \cap \operatorname{Supp}\left(a-a^{\prime}\right) \neq \emptyset$. This distance generates the topology we have defined, but presenting this space as a metric space would be unnatural, because there is (apparently) no canonical choice of such a distance (it depends on a completely arbitrary enumeration of $\Delta$ ).

A last interesting observation is that the subspace of $\mathbf{k}\left\langle\mathcal{N}_{1}\right\rangle$ spanned by the normal resource term is linearly compact, so that NF can be seen as a projection onto a linearly compact subspace.

\subsection{Dealing with free variables}

The finiteness space $\mathcal{N}_{1}$ allows to give meaning to normalization as shown by Proposition 8 , but we would also like to deal with elements of $\mathcal{N}_{1}$ (or of $\mathbf{k}\left\langle\mathcal{N}_{1}\right\rangle$ ) as if they were lambda-terms. However, nothing prevents an element $e$ of $\mathcal{N}_{1}$ of containing infinitely many free variables. The set $\mathrm{FV}(e)$ can even be the set of all variables: take for $e$ the set of all variables itself! It would be hard to define $\beta$-reduction if we have to deal with such objects.

Fortunately the solution to this problem is quite easy. Let $\mathcal{S} \subseteq \Delta$ be the set of all subsets $e^{\prime}$ of $\Delta$ such that, for each finite set $\xi$ of variables, there are only finitely many elements $s$ of $e^{\prime}$ such that $\mathrm{FV}(s) \subseteq \xi$.

Lemma $11 \mathcal{S}^{\perp}=\{e \subseteq \Delta \mid \mathrm{FV}(e)$ is finite $\}$

Proof. The inclusion " $\supseteq$ " is straightforward. So let $e \in \mathcal{S}^{\perp}$. Towards a contradiction, assume that FV $(e)$ is infinite and let $x_{1}, x_{2} \ldots$ be a repetition-free enumeration of this set of variables. Let $n_{1}=1$. Choose $s_{1} \in e$ such that $x_{1} \in \mathrm{FV}\left(s_{1}\right)$. Since $\mathrm{FV}\left(s_{1}\right)$ is finite, we can find $n_{2}$ such that $\mathrm{FV}\left(s_{1}\right) \cap\left\{x_{i} \mid i \geq n_{2}\right\}=\emptyset$. Choose $s_{2} \in e$ such that $x_{n_{2}} \in \mathrm{FV}\left(s_{2}\right)$, choose $n_{3}$ such that $\mathrm{FV}\left(s_{2}\right) \cap\left\{x_{i} \mid i \geq n_{3}\right\}=\emptyset \ldots$ In that way we define a sequence $s_{1}, s_{2}, \ldots$ of element of $e$ and a sequence $y_{1}, y_{2}, \ldots$ of variables such that $y_{i} \in \mathrm{FV}\left(s_{j}\right)$ iff $i=j$ (take $y_{i}=x_{n_{i}}$ ). Then $e^{\prime}=\left\{s_{i} \mid i=1,2, \ldots\right\}$ is an element of $\mathcal{S}$. Indeed, if $\xi$ is a finite set of variables, $\xi$ contains only a finite number of $y_{i}$ 's and hence there can be only finitely many $i$ 's such that $\mathrm{FV}\left(s_{i}\right) \subseteq \xi$. But $e \cap e^{\prime}$ is infinite since $e^{\prime} \subseteq e$, whence the contradiction.

This is another instance of a general proof scheme used several times in [Ehr05] and generalized by Tasson and Vaux (see [Tas09]).

We arrive to the final definition of our basic finiteness space: we set $\mathcal{N}=\mathcal{N}_{1} \cap \mathcal{S}^{\perp}=(\{\uparrow s \mid s \in \Delta\} \cup \mathcal{S})^{\perp}$ and therefore we have $\mathcal{N}^{\perp \perp}=\mathcal{N}$ so that $\mathcal{N}$ is actually a finiteness space.

\section{Interpreting types}

With any type (of system F, see Section 5.1), we want to associate a finiteness space whose web will be a subset of $\Delta$. The construction is based on the definition of saturated sets in [Kri93], so we shall call our finiteness spaces saturated as well.

Let $\mathcal{N}_{0}$ be the collection of all subsets of $\Delta$ which are of the shape $\left\langle\left\langle\langle x\rangle e_{1} !\right\rangle \cdots\right\rangle e_{n} !$ where $x$ is a variable and $e_{1}, \ldots, e_{n} \in \mathcal{N}$.

\subsection{Saturated finiteness space}

A $\Delta$-finiteness space is a finiteness space $X$ such that $|X| \subseteq \Delta$. One says that such a space $X$ is saturated if $\mathcal{N}_{0} \subseteq \mathrm{F}(X) \subseteq \mathcal{N}$ and, whenever $g, e, e_{1}, \ldots, e_{n} \in \mathcal{N}$, one has (using the notations introduced in 1.1.1 and 1.1.2) the implication

$$
\begin{aligned}
& \left\langle\left\langle\left\langle\partial_{x}\left(g, e^{!}\right)\right\rangle e_{1}{ }^{\dagger}\right\rangle \cdots\right\rangle e_{n}{ }^{!} \in \mathrm{F}(X) \\
& \Rightarrow\left\langle\left\langle\left\langle\langle\lambda x g\rangle e^{!}\right\rangle e_{1}^{!}\right\rangle \cdots\right\rangle e_{n} ! \in \mathrm{F}(X) .
\end{aligned}
$$

\footnotetext{
${ }^{7}$ This notion is defined in [Lef42]; it is a notion of compactness adapted to this setting.
} 
Then one simply says that $X$ is a saturated finiteness space.

Given two $\Delta$-finiteness spaces $X$ and $Y$, we construct a new one, denoted as $X \Rightarrow Y$.

The web $|X \Rightarrow Y|$ is the collection of all $t \in \Delta$ such that

$$
\forall e \in \mathrm{F}(X) \quad\langle t\rangle e^{!} \in \mathrm{F}(Y) .
$$

Then we define $\mathrm{F}(X \Rightarrow Y)$ as the collection of all $g \subseteq|X \Rightarrow Y|$ such that

$$
\forall e \in \mathrm{F}(X) \quad\langle g\rangle e^{!} \in \mathrm{F}(Y),
$$

that is

$$
\forall e \in \mathrm{F}(X), \forall f^{\prime} \in \mathrm{F}(Y)^{\perp} \quad\langle g\rangle e^{!} \cap f^{\prime} \text { is finite. }
$$

Given $e \in \mathrm{F}(X)$ and $f^{\prime} \in \mathrm{F}(Y)^{\perp}$, let $e \bullet f^{\prime}=\left\{t \in \Delta \mid\langle t\rangle e^{!} \cap f^{\prime} \neq \emptyset\right\}$.

Proposition 12 If $X$ and $Y$ are $\Delta$-finiteness spaces, then

$$
\mathrm{F}(X \Rightarrow Y)=\left\{e \bullet f^{\prime} \mid e \in \mathrm{F}(X), f^{\prime} \in \mathrm{F}(Y)^{\perp}\right\}^{\perp}
$$

so that $X \Rightarrow Y$ is a $\Delta$-finiteness space. If moreover $Y$ is saturated, then $X \Rightarrow Y$ is saturated as well.

Proof. Let us check equation (3), so let $g \subseteq|X \Rightarrow Y|$.

Assume first that $g \in \mathrm{F}(X \Rightarrow Y)$. Let $e \in \mathrm{F}(X)$ and $f^{\prime} \in \mathrm{F}(Y)^{\perp}$. We know that $\langle g\rangle e^{!} \cap f^{\prime}$ is finite. Let $t \in g \cap\left(e \bullet f^{\prime}\right)$. This means that there exists $S_{t} \in e^{!}$such that $\langle t\rangle S_{t} \in f^{\prime}$, that is, $\langle t\rangle S_{t} \in\langle g\rangle e^{!} \cap f^{\prime}$. But this latter set is finite, and the map $t \mapsto\langle t\rangle S_{t}$ is injective, so the set $g \cap\left(e \bullet f^{\prime}\right)$ is finite as well.

Assume that $g \in\left\{e \bullet f^{\prime} \mid e \in \mathrm{F}(X) \text { and } f^{\prime} \in \mathrm{F}(Y)^{\perp}\right\}^{\perp}$ and let us show that $g \in \mathrm{F}(X \Rightarrow Y)$. So let $e \in \mathrm{F}(X)$ and $f^{\prime} \in \mathrm{F}(Y)^{\perp}$, we must show that $\langle g\rangle e^{!} \cap f^{\prime}$ is finite. By definition of $e \bullet f^{\prime}$, we have

$$
\langle g\rangle e^{!} \cap f^{\prime}=\bigcup_{t \in g \cap\left(e \bullet f^{\prime}\right)}\left(\langle t\rangle e^{!} \cap f^{\prime}\right)
$$

and we conclude since $g \cap\left(e \bullet f^{\prime}\right)$ is finite, and, for $t \in g$, the set $\langle t\rangle e^{!} \cap f^{\prime}$ is finite since $g \subseteq|X \Rightarrow Y|$ (remember the definition above of that set).

So $X \Rightarrow Y=(|X \Rightarrow Y|, \mathrm{F}(X \Rightarrow Y))$ is a finiteness space. Assume that $Y$ is saturated and let us show that $X \Rightarrow Y$ is.

We have $\mathcal{N}_{0} \subseteq \mathrm{F}(X \Rightarrow Y)$ : this results immediately from $\mathcal{N}_{0} \subseteq \mathrm{F}(Y)$ and $\mathrm{F}(X) \subseteq \mathcal{N}$.

We have $\mathrm{F}(X \Rightarrow Y) \subseteq \mathcal{N}$ : let $g \in \mathrm{F}(X \Rightarrow Y)$ and let $t \in \Delta$. We must show that $g \cap \uparrow t$ is finite, so assume towards a contradiction that there are $t_{1}, t_{2}, \cdots \in g$, pairwise distinct, and such that $t_{i} \in \uparrow t$ for each $i$. This means that there are terms $a_{1}, a_{2}, \cdots \in \mathbb{N}\langle\Delta\rangle$ such that $t_{i} \beta_{\Delta} a_{i}$ and $t \in a_{i}$ for each $i$. Let $x$ be an arbitrary variable, then $\left\langle t_{i}\right\rangle x \beta_{\Delta}\left\langle a_{i}\right\rangle x$ and $\langle t\rangle x \in \operatorname{Supp}\left(\left\langle a_{i}\right\rangle x\right)$ for each $i$, therefore $\langle g\rangle x \cap \uparrow\langle t\rangle x$ is infinite, which is impossible because $\{x\} \in \mathrm{F}(X)$ (since $\left.\mathcal{N}_{0} \subseteq|X|\right)$ and $\mathrm{F}(Y) \subseteq \mathcal{N}$.

It remains to check that $\mathrm{F}(X \Rightarrow Y)$ satisfies condition (2), and this is straightforward.

\subsection{The ground space}

Lemma 13 The finiteness space $(\Delta, \mathcal{N})$ is saturated. 
Proof. The only condition which is not obviously satisfied is (2). So let $g, e, e_{1}, \ldots, e_{n} \in \mathcal{N}$ and assume that $\left\langle\left\langle\left\langle\partial_{x}\left(g, e^{!}\right)\right\rangle e_{1}{ }^{!}\right\rangle \cdots\right\rangle e_{n}{ }^{!} \in \mathcal{N}$. Let $s \in \Delta$, we must show that the intersection $\uparrow s \cap\left\langle\left\langle\left\langle\langle\lambda x g\rangle e^{!}\right\rangle e_{1}{ }^{!}\right\rangle \cdots\right\rangle e_{n}{ }^{!}$is finite. Let $\left(s_{i}, S_{i}, S_{1, i}, \ldots, S_{n, i}\right)_{i \in I}$ be a repetition free enumeration of all the elements of $g \times e^{!} \times e_{1} ! \times \cdots \times e_{n} !$ such that

$$
\begin{aligned}
t_{i}=\left\langle\left\langle\left\langle\left\langle\lambda x s_{i}\right\rangle S_{i}\right\rangle S_{1, i}\right\rangle \cdots\right\rangle S_{n, i} \\
\in \uparrow s \cap\left\langle\left\langle\left\langle\langle\lambda x g\rangle e^{!}\right\rangle e_{1}{ }^{!}\right\rangle \cdots\right\rangle e_{n} !
\end{aligned}
$$

Observe that all the free variables of the terms $t_{i}$ appear free in $s$ and hence there are only finitely many such variables. So we can choose a variable $y$ which is free in none of these terms. For each $i \in I$, we set $v_{i}=\left\langle\left\langle\langle y\rangle S_{1, i}\right\rangle \cdots\right\rangle S_{n, i} \in \Delta$, so that $t_{i}=v_{i}\left[\left\langle\lambda x s_{i}\right\rangle S_{i} / y\right]$. We can also assume that $x$ occurs free or bound in none of the terms $S_{i}, S_{1, i}, \ldots, S_{n, i}$ (for all $i \in I$ ). We apply Lemma [5, considering two cases.

- Either $x$ appears bound in $s$, and in that case we have $s=v^{\prime}\left[\left\langle\lambda x s^{\prime}\right\rangle S^{\prime} / y\right]$ for some $v^{\prime}, s^{\prime} \in \Delta$ and $S^{\prime} \in \Delta^{!}$ such that $v^{\prime} \leq v_{i}, s^{\prime} \leq s_{i}$ and $S^{\prime} \leq S_{i}$ for each $i \in I$. We have $v^{\prime}=\left\langle\left\langle\langle y\rangle S_{1}^{\prime}\right\rangle \cdots\right\rangle S_{n}^{\prime}$ for $S_{1}^{\prime}, \ldots, S_{n}^{\prime} \in \Delta^{!}$such that $S_{j}^{\prime} \leq S_{j, i}$ for each $j \in\{1, \ldots, n\}$ and $i \in I$. By the assumption that $g, e, e_{1}, \ldots, e_{n} \in \mathcal{N}$ we see that the sets $\left\{s_{i} \mid i \in I\right\},\left\{S_{i} \mid i \in I\right\},\left\{S_{1, i} \mid i \in I\right\}, \ldots,\left\{S_{n, i} \mid i \in I\right\}$ are finite and so $\uparrow s \cap\left\langle\left\langle\left\langle\langle\lambda x g\rangle e^{!}\right\rangle e_{1}{ }^{!}\right\rangle \cdots\right\rangle e_{n} !$ is finite.

- Or $x$ does not appear bound in $s$. Then for each $i \in I$ there exists $u_{i} \in \Delta$ such that $u_{i} \in \partial_{x}\left(s_{i}, S_{i}\right)$ and $s \leq v_{i}\left[u_{i} / y\right]$. In other words

$$
\forall i \in I \quad v_{i}\left[u_{i} / y\right] \in \uparrow s \cap\left\langle\left\langle\left\langle\partial_{x}\left(g, e^{!}\right)\right\rangle e_{1}{ }^{!}\right\rangle \cdots\right\rangle e_{n}{ }^{!}
$$

and hence by our assumption that $\left\langle\left\langle\left\langle\partial_{x}\left(g, e^{!}\right)\right\rangle e_{1}{ }^{!}\right\rangle \cdots\right\rangle e_{n}{ }^{!} \in \mathcal{N}$, the set $\left\{v_{i}\left[u_{i} / y\right] \mid i \in I\right\}$ is finite. Coming back to the definition of $v_{i}$, this means that the sets $\left\{u_{i} \mid i \in I\right\},\left\{S_{1, i} \mid i \in I\right\}, \ldots,\left\{S_{n, i} \mid i \in I\right\}$ are finite. But for each $i \in I$, we know that there are only finitely many pairs $(w, W) \in \Delta \times \Delta^{!}$such that $u_{i} \in \partial_{x}(w, W)$ by Lemma 6 and hence, since $u_{i} \in \partial_{x}\left(s_{i}, S_{i}\right)$, the sets $\left\{s_{i} \mid i \in I\right\}$ and $\left\{S_{i} \mid i \in I\right\}$ must be finite as well since $\left\{s_{i} \mid i \in I\right\}$ is finite.

\subsection{Inclusions and intersections of saturated finiteness spaces}

Let $X$ and $Y$ be saturated finiteness spaces. We write $X \subseteq Y$ when $|X| \subseteq|Y|$ and $\mathrm{F}(X) \subseteq \mathrm{F}(Y)$. This defines an order relation on saturated finiteness spaces.

Lemma 14 Let $\left(X_{i}\right)_{i \in I}$ be a family of saturated finiteness spaces. Then $\bigcap_{i \in I} X_{i}=\left(\bigcap_{i \in I}\left|X_{i}\right|, \bigcap_{i \in I}\left(\mathrm{~F}\left(X_{i}\right) \cap\right.\right.$ $\left.\left.\mathcal{P}\left(|X|_{i}\right)\right)\right)$ is a saturated finiteness space, and it is le glb of the family $\left(X_{i}\right)_{i \in I}$.

Proof. Let $X=\bigcap_{i \in I} X_{i}$. Let $e \subseteq|X|=\bigcap_{i \in I}\left|X_{i}\right|$. We assume that $e \in \mathrm{F}(X)^{\perp \perp}$ and we prove that $e \in \mathrm{F}(X)$. Let $i \in I$, we must show that $e \in \mathrm{F}\left(X_{i}\right)=\mathrm{F}\left(X_{i}\right)^{\perp \perp}$. So let $e^{\prime} \subseteq\left|X_{i}\right|$ and let us show that $e \cap e^{\prime}$ is finite. Since $e \in \mathrm{F}(X)^{\perp \perp}$, it will be sufficient to show that $e^{\prime} \in \mathrm{F}(X)^{\perp}$. So let $f \subseteq|X|$ be such that $f \in \mathrm{F}(X)$. In particular we have $f \in \mathrm{F}\left(X_{i}\right)$ and hence $e^{\prime} \cap f$ is finite as required. So $X$ is a $\Delta$-finiteness space.

Since $\mathcal{N}_{0} \subseteq \mathrm{F}\left(X_{i}\right) \subseteq \mathcal{N}$ holds for all $i \in I$, and since $I$ is non empty, it is clear that $\mathcal{N}_{0} \subseteq \mathrm{F}(X) \subseteq \mathcal{N}$.

Let $g, e, e_{1}, \ldots, e_{n} \in \mathcal{N}$ be such that $\left\langle\left\langle\left\langle\partial_{x}\left(g, e^{!}\right)\right\rangle e_{1}{ }^{!}\right\rangle \cdots\right\rangle e_{n} ! \in \mathrm{F}(X)$. Then for each $i$ we have $\left\langle\left\langle\left\langle\partial_{x}\left(g, e^{!}\right)\right\rangle e_{1}{ }^{!}\right\rangle \cdots\right\rangle e_{n} ! \in$ $\mathrm{F}\left(X_{i}\right)$ and hence $\left\langle\left\langle\left\langle\langle\lambda x g\rangle e^{!}\right\rangle e_{1}{ }^{!}\right\rangle \cdots\right\rangle e_{n}{ }^{!} \in \mathrm{F}\left(X_{i}\right)$ and therefore $\left\langle\left\langle\left\langle\langle\lambda x g\rangle e^{!}\right\rangle e_{1}{ }^{!}\right\rangle \cdots\right\rangle e_{n}{ }^{!} \in \mathrm{F}(X)$.

\section{Taylor expansion in an algebraic system $\mathbf{F}$}

\subsection{Syntax of the algebraic system $F$}

The types are defined as usual: one has type variables $\varphi, \psi \ldots$, and if $A$ and $B$ are types, so are $A \Rightarrow B$ and $\forall \varphi A$. We adopt the Curry style for presenting system F, so that our terms are ordinary lambda-terms, with the additional possibility of linearly combining terms, with coefficients in $\mathbf{k}$. More precisely, we define the set $\Lambda_{\mathbf{k}}$ of lambda-terms with coefficients in $\mathbf{k}$ as follows: 
- if $x$ is a variable then $x \in \Lambda_{\mathbf{k}}$;

- if $M \in \Lambda_{\mathbf{k}}$ and $x$ is a variable, then $\lambda x M \in \Lambda_{\mathbf{k}}$;

- if $M \in \Lambda_{\mathbf{k}}$ and $Q \in \mathbf{k}\left\langle\Lambda_{\mathbf{k}}\right\rangle$ then $(M) Q \in \Lambda_{\mathbf{k}}$.

For $Q, R \in \mathbf{k}\left\langle\Lambda_{\mathbf{k}}\right\rangle$, we set $\lambda x Q=\sum_{M \in \Lambda_{\mathbf{k}}} Q_{M} \lambda x M$ and $(Q) R=\sum_{M \in \Lambda_{\mathbf{k}}} Q_{M}(M) R$. Observe that these two sums are finite because $Q$ is a finite linear combination of terms. In other word, abstraction is linear and application is left-linear (but not right-linear). We give now the typing rules for terms belonging to $\Lambda_{\mathbf{k}}$. A typing context $\Gamma$ is as usual a finite partial function from variables to types.

$$
\begin{array}{cc}
\frac{\Gamma, x: A \vdash M: B}{\Gamma, x: A \vdash x: A} & \frac{\Gamma \vdash \lambda x M: A \Rightarrow B}{\Gamma \vdash x} \\
\frac{\Gamma \vdash M: A \Rightarrow B \quad \Gamma \vdash N_{1}: A \quad \ldots \quad \Gamma \vdash N_{n}: A}{\Gamma \vdash(M)\left(\alpha_{1} N_{1}+\cdots+\alpha_{n} N_{n}\right): B} \\
\frac{\Gamma \vdash M: \forall \varphi A}{\Gamma \vdash M: A[B / \varphi]} \quad \frac{\Gamma \vdash M: A}{\Gamma \vdash M: \forall \varphi A}
\end{array}
$$

with, for the last rule, the usual side condition that $\varphi$ should not occur free in the typing context $\Gamma$.

\subsection{Taylor expansion}

Given a term $M \in \Lambda_{\mathbf{k}}$ (resp. $Q \in \mathbf{k}\left\langle\Lambda_{\mathbf{k}}\right\rangle$ ), we define a generally infinite linear combinations $M^{*}$ (resp. $Q^{*}$ ) of elements of $\Delta$, with coefficients in $\mathbf{k}$, as follows:

$$
\begin{aligned}
x^{*} & =x \\
(\lambda x M)^{*} & =\lambda x\left(M^{*}\right) \\
((M) Q)^{*} & =\sum_{n \in \mathbb{N}} \frac{1}{n !}\left\langle M^{*}\right\rangle\left(Q^{*}\right)^{n} \\
Q^{*} & =\sum_{M \in \Lambda_{\mathbf{k}}} Q_{M} Q^{*}
\end{aligned}
$$

where we use the conventions of 1.1 .1 for infinite linear combinations of terms. Let us be more explicit. With any term $M \in \Lambda_{\mathbf{k}}$, we associate a linear combination $M^{*}$ of elements of $\Delta$ which can be written

$$
M^{*}=\sum_{s \in \Delta} M_{s}^{*} s
$$

where $M_{s}^{*} \in \mathbf{k}$ for each $s$, and similarly we define $Q_{s}^{*} \in \mathbf{k}$ for each $Q \in \mathbf{k}\left\langle\Lambda_{\mathbf{k}}\right\rangle$. Then these numbers are given inductively by:

$$
\begin{aligned}
x_{s}^{*} & = \begin{cases}1 & \text { if } s=x \\
0 & \text { otherwise }\end{cases} \\
(\lambda x M)_{s}^{*} & = \begin{cases}M_{t}^{*} & \text { if } s=\lambda x t \\
0 & \text { otherwise }\end{cases} \\
Q_{s}^{*} & =\sum_{M \in \Lambda_{\mathbf{k}}} Q_{M} M_{s}^{*}
\end{aligned}
$$


Last, $((M) Q)_{s}^{*}=0$ if $s$ is not an application, and otherwise

$$
\begin{aligned}
((M) Q)_{\langle t\rangle T}^{*} & =\left(\sum_{n \in \mathbb{N}} \frac{1}{n !}\left\langle M^{*}\right\rangle\left(Q^{*}\right)^{n}\right)_{\langle t\rangle T} \\
& =\sum_{n \in \mathbb{N}} \frac{M_{t}^{*}}{n !}\left(Q^{*}\right)_{T}^{n} \\
& =\sum_{n \in \mathbb{N}} \frac{M_{t}^{*}}{n !}\left(\sum_{u \in \Delta} Q_{u}^{*} u\right)_{T}^{n} \\
& =\frac{M_{t}^{*}\left(Q^{*}\right)^{T}}{T !}
\end{aligned}
$$

where $T !=\prod_{u \in \Delta} T(u)$ ! and $\left(Q^{*}\right)^{T}=\prod_{u \in \Delta}\left(Q_{u}^{*}\right)^{T(u)}$ (see [ER08] for more details on this kind of algebraic computations); remember that $T$ is a finite multiset of elements of $\Delta$ and that $T(u) \in \mathbb{N}$ is the multiplicity of $u$ in $T$.

Given $M \in \Lambda_{\mathbf{k}}$, we define a set $\mathcal{T}(M) \subseteq \Delta$ as follows:

$$
\begin{aligned}
& \mathcal{T}(x)=\{x\}, \quad \mathcal{T}(\lambda x M)=\{\lambda x s \mid s \in \mathcal{T}(M)\} \\
& \mathcal{T}\left((M)\left(\alpha_{1} N_{1}+\cdots+\alpha_{n} N_{n}\right)\right) \\
& =\left\{\langle s\rangle\left(t_{1} \cdots t_{p}\right) \mid s \in \mathcal{T}(M)\right. \\
& \left.\quad \quad \text { and } t_{1}, \ldots, t_{p} \in \mathcal{T}\left(N_{1}\right) \cup \cdots \cup \mathcal{T}\left(N_{n}\right)\right\} .
\end{aligned}
$$

The following property follows readily from these definitions.

Lemma 15 Let $M \in \Lambda_{\mathbf{k}}$ and $s \in \Delta$. If $M_{s}^{*} \neq 0$ then $s \in \mathcal{T}(M)$.

\subsection{The standard case: coherence}

When the algebraic lambda-term $M$ is a standard lambda-term, that is an element of $\Lambda_{\mathbf{k}}$ where all the linear combinations $\alpha_{1} N_{1}+\cdots+\alpha_{p} N_{p}$ are trivial in the sense that all $\alpha_{i}$ 's are equal to 0 but one which is equal to 1 , we showed in [ER08] that the Taylor expansion can be written $M^{*}=\sum_{s \in \mathcal{T}(M)} \frac{1}{m(s)} s$ where $m(s) \in \mathbb{N} \backslash\{0\}$ is an integer which depends only on $s$ (in other words $M_{s}^{*}$ depends on $M$ in a very simple way: $M_{s}^{*}=0$ if $s \notin \mathcal{T}(M)$, and otherwise $M_{s}^{*}=1 / m(s)$ ). Moreover the various elements of $\mathcal{T}(M)$ cannot overlap during their reduction, in the sense that if $s, t \in \mathcal{T}(M)$ are distinct then $\mathrm{NF}(s) \cap \mathrm{NF}(t)=\emptyset$. This is proven by introducing a binary symmetric but not reflexive coherence relation, observing that each set $\mathcal{T}(M)$ is a clique for this coherence relation and proving that $\mathrm{NF}$ can be seen as a stable and linear function on this coherence space (in the sense of [Gir86]).

These properties are lost in the present setting and superpositions can occur and even lead to infinite sums, as in the Taylor expansion (that we do not compute here) of the term $M=(\Theta) \lambda x(x+z)$ where $z$ is a variable $\neq x$ and $\Theta$ is the Turing fixpoint combinator (reducing $M$ leads to terms of the shape $n z+M$ for all $n \in \mathbb{N}$ ). This superposition of elementary normal forms is controlled by the finiteness structures, but this is possible only in a typed setting (here, second order types).

\subsection{Finiteness of the Taylor expansions in system $\mathbf{F}$}

\subsubsection{Interpreting types}

A type valuation is a map $\mathcal{I}$ which associates a saturated finiteness space $\mathcal{I}(\varphi)$ with any type variable $\varphi$. By induction on type $A$ we define, for all valuation $\mathcal{I}$, a saturated finiteness space $[A]^{\mathcal{I}}$ in a fairly standard way: $[\varphi]^{\mathcal{I}}=\mathcal{I}(\varphi)$, $[(A \Rightarrow B)]^{\mathcal{I}}=[A]^{\mathcal{I}} \Rightarrow[B]^{\mathcal{I}}$ and $[(\forall \varphi A)]^{\mathcal{I}}=\bigcap_{X \in \mathrm{SFS}}[A]^{\mathcal{I}[\varphi \mapsto X]}$ where SFS is the class of all saturated finiteness spaces (remember that the intersection of saturated finiteness spaces is defined in Section 4.3). 


\subsubsection{The fundamental property}

Our goal is to prove that, if $\Gamma \vdash M: A$, then $\mathcal{T}(M) \in \mathrm{F}\left([A]^{\mathcal{I}}\right)$ for any valuation $\mathcal{I}$. Of course this property cannot be proven in that form and a more general statement is needed.

Proposition 16 Let $\Gamma=\left(x_{1}: A_{1}, \ldots, x_{n}: A_{n}\right)$ be a typing context. Assume that $\Gamma \vdash M: B$, where $M \in \Lambda_{\mathbf{k}}$ and $B$ and the $A_{i}$ 's are second order types. Let $\mathcal{I}$ be a valuation. Let $e_{1} \in \mathrm{F}\left(\left[A_{1}\right]^{\mathcal{I}}\right), \ldots, e_{n} \in \mathrm{F}\left(\left[A_{n}\right]^{\mathcal{I}}\right)$ be sets of simple terms and let $f=\mathcal{T}(M)$. Then $\partial_{x_{1}, \ldots, x_{n}}\left(f, e_{1}{ }^{!}, \ldots, e_{n}{ }^{!}\right) \in \mathrm{F}\left([B]^{\mathcal{I}}\right)$.

Proof. Adaptation from the proof of strong normalization of system F in [Kri93], see the Appendix.

By Lemma 15, this shows in particular that, if $M \in \Lambda_{\mathbf{k}}$ is typeable in system F, then $M^{*} \in \mathbf{k}\langle\mathcal{N}\rangle$ so that we can reduce the infinitely many resource terms appearing in this expansion without creating any infinite superimposition of terms, whatever be the choices we make in this process. Of course, one can also prove that $\operatorname{NF}\left(M^{*}\right)=M_{0}{ }^{*}$ where $M_{0}$ is the normal form of $M$, but this is not straightforward.

\section{Conclusion}

Following the line of ideas initiated in [ER03, ER08, ER06a], we considered the resource lambda-calculus as an algebraic setting where various (algebraic, differential...) extensions of the lambda-calculus can be interpreted. In this setting, the elementary points of the interpretation (the simple resource terms) are considered as base vectors and, in sharp contrast with denotational semantics, have their own completely finite dynamics. We introduced topologies for controlling their global behavior during reduction and avoiding the appearance of infinite coefficients: linear combinations of resource terms are organized as Hausdorff and complete topological vector spaces associated with types. By a rather standard reducibility argument, we proved that the Taylor expansion of any term of an algebraic extension of system $\mathrm{F}$ belongs to the vector space interpretation of its type, but of course these vector spaces contain many elements which are not Taylor expansions of such terms.

For instance, given $a \in \mathbf{k}\langle X \Rightarrow Y\rangle$, it is not difficult to define $a^{\prime} \in \mathbf{k}\langle X \Rightarrow(X \Rightarrow Y)\rangle$, the derivative of $a$ (which is linear in its first parameter of type $X$ ). Saying that $a^{\prime}$ is linear means that $\left\langle a^{\prime}\right\rangle x^{n}=_{\Delta} 0$ for $n \neq 1$, where $x$ is an arbitrary variable. One can show that this operation can be reversed (under a necessary and sufficient condition), so that it makes sense to compute "primitives" of resource terms and it is certainly a fascinating challenge to understand the operational meaning of this operation.

\section{References}

[AD08] Pablo Arrighi and Gilles Dowek. Linear-algebraic lambda-calculus: higher-order, encodings, and confluence. In Andrei Voronkov, editor, RTA, volume 5117 of Lecture Notes in Computer Science, pages 17-31. Springer, 2008.

[BCL99] Gérard Boudol, Pierre-Louis Curien, and Carolina Lavatelli. A semantics for lambda calculi with resource. Mathematical Structures in Computer Science, 9(4):437-482, 1999.

[Bou93] Gérard Boudol. The lambda calculus with multiplicities. Technical Report 2025, INRIA Sophia-Antipolis, 1993.

[Ehr05] Thomas Ehrhard. Finiteness spaces. Mathematical Structures in Computer Science, 15(4):615-646, 2005.

[EL09] Thomas Ehrhard and Olivier Laurent. Interpreting a finitary pi-calculus in differential interaction nets. Information and Computation, 2009. To appear.

[ER03] Thomas Ehrhard and Laurent Regnier. The differential lambda-calculus. Theoretical Computer Science, 309(1-3):1-41, 2003. 
[ER06a] Thomas Ehrhard and Laurent Regnier. Böhm trees, Krivine machine and the Taylor expansion of ordinary lambda-terms. In Arnold Beckmann, Ulrich Berger, Benedikt Löwe, and John V. Tucker, editors, Logical Approaches to Computational Barriers, volume 3988 of Lecture Notes in Computer Science, pages 186197. Springer-Verlag, 2006.

[ER06b] Thomas Ehrhard and Laurent Regnier. Differential interaction nets. Theoretical Computer Science, 364(2):166-195, 2006.

[ER08] Thomas Ehrhard and Laurent Regnier. Uniformity and the Taylor expansion of ordinary lambda-terms. Theoretical Computer Science, 403(2-3):347-372, 2008.

[Gir86] Jean-Yves Girard. The system F of variable types, fifteen years later. Theoretical Computer Science, 45:159-192, 1986.

[Kfo00] Assaf J. Kfoury. A linearization of the lambda-calculus. Journal of Logic and Computation, 10(3):411-436, 2000.

[Kri93] Jean-Louis Krivine. Lambda-Calculus, Types and Models. Ellis Horwood Series in Computers and Their Applications. Ellis Horwood, 1993. Translation by René Cori from French 1990 edition (Masson).

[Lef42] Solomon Lefschetz. Algebraic topology. Number 27 in American mathematical society colloquium publications. American Mathematical Society, 1942.

[PT09a] Michele Pagani and Christine Tasson. The Taylor Expansion Inverse problem in Linear Logic. In Proceedings of the 24th Annual IEEE Symposium on Logic in Computer Science, pages 222-232. IEEE Computer Society, 2009.

[PT09b] Michele Pagani and Paolo Tranquilli. Parallel Reduction in Resource Lambda-Calculus. In Zhenjiang Hu, editor, APLAS, volume 5904 of Lecture Notes in Computer Science, pages 226-242. Springer, 2009.

[Tas09] Christine Tasson. Sémantiques et syntaxes vectorielles de la logique linéaire. Thèse de doctorat, Université Paris Diderot - Paris 7, 2009.

[Tra08] Paolo Tranquilli. Intuitionistic Differential Nets and Lambda-Calculus. Theoretical Computer Science, 2008. To appear.

[Vau05] Lionel Vaux. The differential lambda-mu calculus. Theoretical Computer Science, 379(1-2):166-209, 2005.

[Vau07] Lionel Vaux. On linear combinations of lambda-terms. In Term Rewriting and Applications, volume 4533 of Lecture Notes in Computer Science, pages 374-388. Springer-Verlag, 2007.

[Vau08] Lionel Vaux. The algebraic lambda-calculus. Mathematical Structures in Computer Science, 2008.

\section{Appendix: proof of proposition 16}

We adopt the following notational convention: if $g \in \mathrm{F}\left([C]^{\mathcal{I}}\right)$ for some type $C$ then we use $g^{\prime}$ to denote the set $\partial_{x_{1}, \ldots, x_{n}}\left(g, e_{1} !, \ldots, e_{n} !\right)$.

The proof is by induction on the typing derivation of $x_{1}: A_{1}, \ldots, x_{n}: A_{n} \vdash M: B$ (the statement that we prove by induction is universally quantified in $\mathcal{I}$ and in the $e_{i}$ 's).

Assume first that $M=x_{i}$ and that the derivation consists of the axiom

$$
\overline{\Gamma \vdash x_{i}: A_{i}}
$$


We have $f=\left\{x_{i}\right\}$ and hence

$$
\begin{aligned}
& \partial_{x_{1}, \ldots, x_{n}}\left(f, e_{1} !, \ldots, e_{n}{ }^{!}\right) \\
& \quad=\cup\left\{\partial_{x_{1}, \ldots, x_{n}}\left(x_{i}, S_{1}, \ldots, S_{n}\right) \mid \forall j S_{j} \in e_{j}{ }^{!}\right\} \\
& \quad=e_{i} \in \mathrm{F}\left(\left[A_{i}\right]^{\mathcal{I}}\right) .
\end{aligned}
$$

Assume that $M=(N)\left(\alpha_{1} L_{1}+\cdots+\alpha_{p} L_{p}\right)$ where $N, L_{1}, \ldots, L_{p} \in \Lambda_{\mathbf{k}}$ and that the derivation ends with

$$
\frac{\Gamma \vdash N: A \Rightarrow B \quad \Gamma \vdash L_{1}: A \ldots \Gamma \vdash L_{p}: A}{\Gamma \vdash(N)\left(\alpha_{1} L_{1}+\cdots+\alpha_{p} L_{p}\right): B}
$$

We set $Q=\alpha_{1} L_{1}+\cdots+\alpha_{p} L_{p} \in \mathbf{k}\left\langle\Lambda_{\mathbf{k}}\right\rangle$ and $h=\mathcal{T}(Q)$.

Let $g=\mathcal{T}(N)$ and let $h_{i}=\mathcal{T}\left(L_{i}\right)$ for $i=1, \ldots, p$. By inductive hypothesis, we have $g^{\prime} \in \mathrm{F}\left([A \Rightarrow B]^{\mathcal{I}}\right)$ and $h_{j}^{\prime} \in \mathrm{F}\left([A]^{\mathcal{I}}\right)$ for $j=1, \ldots, p$. Since $h \subseteq h_{1} \cup \cdots \cup h_{p}$ and hence $h^{\prime} \subseteq h_{1}^{\prime} \cup \cdots \cup h_{p}^{\prime} \in \mathrm{F}\left([A]^{\mathcal{I}}\right)$ (remember from Section 2 that $[A]^{\mathcal{I}}$ is closed under finite unions).

By definition of $[A \Rightarrow B]^{\mathcal{I}}$, we have therefore $\left\langle g^{\prime}\right\rangle\left(h^{\prime}\right)^{!} \in \mathrm{F}\left([B]^{\mathcal{I}}\right)$. Since $f=\mathcal{T}((N) Q)=\langle g\rangle h^{!}$, we have $f^{\prime}=\left\langle g^{\prime}\right\rangle\left(h^{\prime}\right)^{!}$and we conclude for that case.

Assume that $M=\lambda x N$ where $N \in \Lambda_{\mathbf{k}}$ and that the derivation ends with

$$
\frac{\Gamma, x: B \vdash N: C}{\Gamma \vdash \lambda x N: B \Rightarrow C}
$$

so that $A=(B \Rightarrow C)$. Let $g=\mathcal{T}(N)$, we have $f=\lambda x g$ and hence $f^{\prime}=\lambda x g^{\prime}$ (as usual we assume that $x$ is different from all the $x_{i}$ 's and does not occur free in the $e_{i}$ 's; this is possible because $e_{i} \in \mathcal{N}$ and hence $\operatorname{FV}\left(e_{i}\right)$ is finite for each $i$, see Section 3.1) and we must prove that $\lambda x g^{\prime} \in \mathrm{F}\left([B \Rightarrow C]^{\mathcal{I}}\right)$. Let $e \in \mathrm{F}\left([B]^{\mathcal{I}}\right)$, we must prove that $\left\langle\lambda x g^{\prime}\right\rangle e^{!} \in \mathrm{F}\left([C]^{\mathcal{I}}\right)$. Since $[C]^{\mathcal{I}}$ is a saturated finiteness space, it suffices to prove that $\partial_{x}\left(g^{\prime}, e^{!}\right)=$ $\partial_{x_{1}, \ldots, x_{n}, x}\left(g, e_{1} !, \ldots, e_{n} !, e^{!}\right) \in \mathrm{F}\left([C]^{\mathcal{I}}\right)$ and this results from the inductive hypothesis.

Assume that the derivation ends with

$$
\frac{\Gamma \vdash M: \forall \varphi A}{\Gamma \vdash M: A[B / \varphi]}
$$

By inductive hypothesis we have

$$
f^{\prime} \in \mathrm{F}\left([\forall \varphi A]^{\mathcal{I}}\right)=\bigcap_{X \in \mathrm{SFS}}[A]^{\mathcal{I}[\varphi \mapsto X]} \subseteq \mathrm{F}\left([A]^{\mathcal{I}\left[\varphi \mapsto[B]^{\mathcal{I}}\right]}\right)
$$

and we conclude because this finiteness space is $[A[B / \varphi]]^{\mathcal{I}}$ (straightforward proof by induction on types).

Last assume that the proof ends with

$$
\frac{\Gamma \vdash M: A}{\Gamma \vdash M: \forall \varphi A}
$$

and remember that $\varphi$ cannot occur free in $\Gamma$. Given a saturated finiteness space $X$ we set $\mathcal{I}_{X}=\mathcal{I}[\varphi \mapsto X]$. Our assumption on the $e_{i}$ 's is that $e_{i} \in \mathrm{F}\left(\left[B_{i}\right]^{\mathcal{I}}\right)$ for each $i$. Let $X$ be a saturated finiteness space. Since $\varphi$ does not occur free in $\Gamma$, we have $e_{i} \in \mathrm{F}\left(\left[B_{i}\right]^{\mathcal{I}_{X}}\right)$ and hence by the inductive hypothesis we have $f^{\prime} \in \mathrm{F}\left([A]^{\mathcal{I}_{X}}\right)$. Since this holds for each $X$, we have $f^{\prime} \in \mathrm{F}\left([\forall \varphi A]^{\mathcal{I}}\right)$. 